\title{
Multi-functional Baby Bed Design Based on Human Factors Engineering
}

\author{
Lili Liu ${ }^{1, a^{*}}$, Cheng $\mathrm{Ge}^{2, \mathrm{~b}}$ Qian Wang ${ }^{3, \mathrm{c}}$ \\ ${ }^{1}$ Jiangsu Key Laboratory of Large Engineering Equipment Detection and Control, Xuzhou Institute \\ of Technology, Xuzhou, Jiangsu Province, 221000,China \\ 2 Jiangsu Key Laboratory of Large Engineering Equipment Detection and Control, Xuzhou Institute \\ of Technology, Xuzhou, Jiangsu Province, 221000,China \\ ${ }^{3}$ Jiangsu Key Laboratory of Large Engineering Equipment Detection and Control, Xuzhou Institute \\ of Technology, \\ adxwlll@sina.com, bqq513189684@gmail.com, cwangqian_920@126.com
}

Keywords: Human factors engineering. Combined type. Baby bed. Multi-function

Abstract: Based on the market at present the development status of the crib, with people because of the engineering research as the breakthrough point of the design, the existing defects and problems of the crib as a starting point,and designed a combined multifunctional crib. It can not only the conversion of multi-function, but also increase the more bells and whistles, and make it better and more convenient by modern families use to improve the comfortableness after transformation and utilization.

Since China's reform and opening up, the rapid development of the national economy, people's living level gradually improve, people are yearning for a better life, it in daily furniture also can be seen, and requirements on the choice of children is the hope of every family, the child's early small bed became parents consider one of the most important furniture. Aiming at the existing problems existing in the process of baby bed used for investigation and analysis, according to the practical and functional design concept to design, redesign the structure of the crib, and the function extension, expand the function of the chairs and stools, placed in the home at the same time to achieve a variety of purposes, to achieve the purpose of convenient for the user[1].

\section{Design point}

The parents more and more attention to the cultivation of the next generation, especially for infants and young children more care. Parents when buying baby products, more considering the factors such as security, mute-function, practical. Currently on the market of baby bed structure function is relatively single, also cannot completely meet the required for growth in infants[2]. In this article innovation development of multi-functional nursery bed is different from other ordinary baby bed, it has the appearance of more human and more perfect function of nursery. With the development of people's material conditions, increasing demand. Produce a baby bed should have not only technical aesthetics and function is to learn, but also business characteristics, because the economy is a foundation, and then is the taste and spirit. And as a business opportunity, mental emotional into modeling design has become a crib is an important part.

\section{The main problem of the project for the existing product people relationship}

Human factors engineering is a new and rapidly developing interdisciplinary, involving a variety 
of disciplines, such as physiology, psychology, anatomy, management, engineering, system science, science, safety science, environmental science, etc. Application field is very broad. In this article, through the use of the existing crib process analysis and research to the user, identified the following issues, and to solve these problems as a multi-purpose crib design important goals[3,4].

First of all, from the psychology Angle consideration, currently on the market generally crib form a single, lack of fashion sense, from the senses is very easy to produce visual fatigue, thus virtually reduced the work efficiency.

Secondly, from the consideration on the existing function of the crib, single structure, the lack of humanization design, even if also appeared on the market at present multi-function baby bed, good for multi-function transformation, but the transformation form the same, the lack of new idea. Therefore, we can consider in the design in increasing transformation of functions as well as adding more bells and whistles, make it more effectively meet the needs of the family.

Finally, from a health point of view, at present, most of the crib design on the market only has the function of sleep is no better from the perspective of consumer health, such as the choice of materials, comfort, can improve the quality of sleep, when converted to seat can let users in the use of time to make it more healthy, more human[5].

\section{Multi-functional design points of the crib}

\section{The use of the crib}

Figure 1 is a normal baby bed under the way of use, inherited the characteristics of the traditional the cradle of the crib, surrounded by the type of bird's nest. The baby is sleeping of time can also be realized in the mother's belly feeling, also is the parcel, resulting in a sense of security, improving the quality of the baby sleep. Figure 2 is the crib conversion seat after use, so that people sit in the half open seats, feet can be put on the pedal, making the body more free and comfortable, but also create a personal illicit close space, more human.

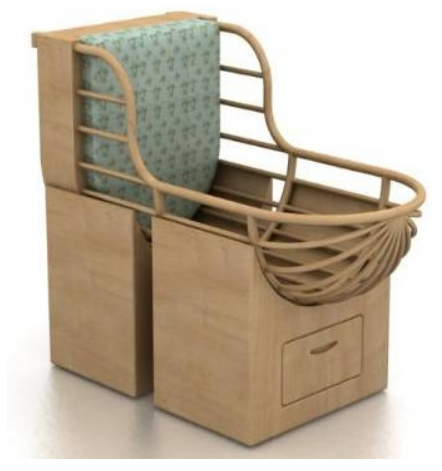

Figure 1 baby bed using

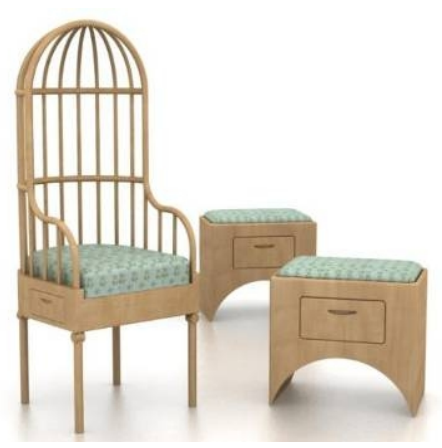

Figure 2 seat state of use

\section{The foot of the bed of the hidden way}

Hidden folding design based on the foot of the bed, not only to reduce the waste of space, but also reduces the unsafe hidden trouble. In addition, look from the Angle of the design, make the appearance more beautiful, the conversion is also more convenient. As shown in figure 3. 


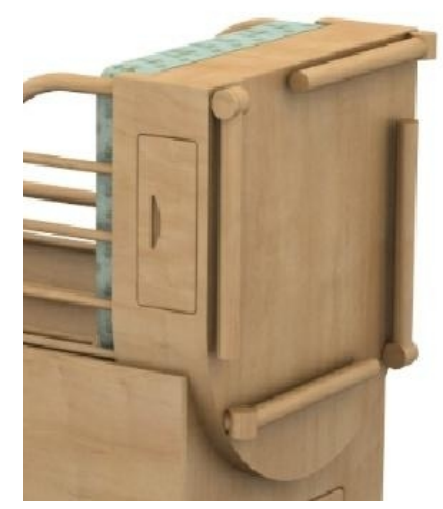

Figure 3 seat legs in

\section{Receive mode}

Through the details of the design, add a drawer to receive a function makes the whole design more rational and functional, believe that such a new design to give new life to this baby bed, more human, more practical. As shown in figure 3, figure 4.

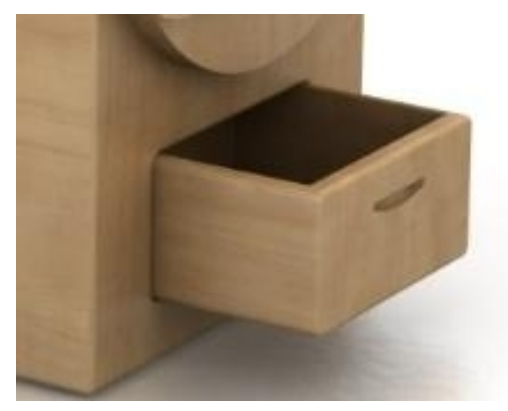

Figure 4 storage drawer

\section{Experience}

This multi-functional baby bed, remove the function, using the existing popular contracted design style, make the whole design is simple and easy. When used as a baby bed, this design of Nest type more sweet feeling and comfort of the children. In addition, when converted to seat, let users can easily comfortable enjoy half open seat novel feeling, also can bring a feeling of encirclement type private and comfort.

\section{Human factors analysis}

\section{Dimensions}

According to the specific condition of the crib design again, which the various components of connection and size must be considered when use of comfort. Will the crib and users and use content composition as a whole system is analyzed, considering the stability of the system, the integrity, aesthetics, etc., make integral harmony[6].

Material size: length $101 \mathrm{~cm}$, the back of the chair seat to chair leg $56 \mathrm{~cm}$ length, the length of the chair seat overall chair height $65 \mathrm{~cm}, 157 \mathrm{~cm}, 60 \mathrm{~cm}$ seat width, half arc radius $31 \mathrm{~cm}$, the back of the chair stool length $60 \mathrm{~cm}$, stools $41 \mathrm{~cm}$ width, bench height of pure $54 \mathrm{~cm}$, pad stool after 58 $\mathrm{cm}$ high. As shown in figure 5, figure 6. 


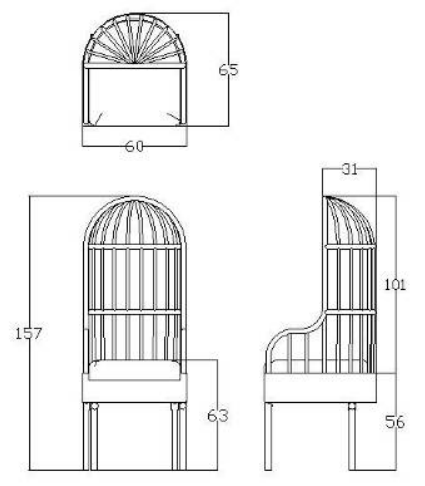

Figure 5 chair size chart
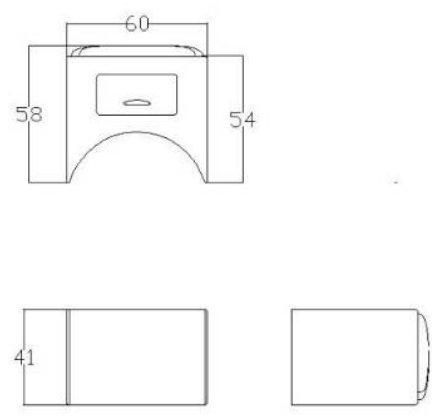

Figure 6 stool size

chart

\section{Material analysis}

Material is the carrier of the final product, how to correctly, reasonably choose materials in the design is a practical and very important problem. Different materials have different characteristics, the most important thing is the material also affect the structure and function of the implementation. For the purpose of this scheme, the main body of the crib should use real wood, because real wood is a natural health does not contain toxic substances. Chairs and stools part choose woodiness material, firm structure, simple sense is good, give a person pure and fresh and natural feeling; Cushion part chooses sponge filling material, soft and comfortable[7].

\section{Color analysis}

Color beautiful visual effect is generally through the fabric produced by this kind of medium to realize and material[8]. The color of the crib design must be specific to the material color conception and creation, through the understanding of materials, the selection and use of the material to express the unique aesthetic feeling and the charm of color, at the same time to the feelings of parents and children, give attention to two or more things color with lively and lively as the main line.

\section{Conclusion}

Baby bed in our country has had the very big development, but the design of new products research and development is always an important topic. The design solves the baby crib bed functional and practical problem for a long time. The innovation point of this design is to cots functionality and long-term practical exploration and bold attempt. Through the combination of chairs and stools clever union, become a warm baby bed, and thus realizes the baby bed and chair stool is practical, and the chair and bench has long-term practical or even permanent practicability. In addition, pay attention to people's physical and mental factors, to accord with the condition of humanized design of human factors engineering, it pay more attention to people's demands as well as the use of comfort for your health. Therefore, in the "people-oriented" design concept, under the guidance of humanization design is not only the need of social development but also the development trend of the future design.

\section{References:}

[1]Zhang Xianrong etc. industrial design theory and method [M].Beijing: Beijing university of science and technology press, 1996. 
[2] Wang Mingzhi. Product design [M].Beijing: China youth publishing house,2002.

[3] TongHuiMing. Designed for life [M].Beijing: China fine arts publishing house, 1998.

[4] Zheng Jianqi Li xiang. Design methodology [M].Beijing: tsinghua university press,2006.

[5] (DE) bernhard.E.Boolean dirk.Industrial design, product modeling history. Theory and practice.Hu Fei ze [J].Beijing: China building industry press, 2007.

[6] Tang Yong medium. Modular fixture assembly technology [M].Beijing: national defense industry press, 1979.

[7] Li Yanzu. Introduction to art and design [M].Wuhan: hubei fine arts publishing house,2001.

[8] yu-lan ding. The man-machine engineering [M].Beijing: Beijing university of science and technology press, 2005. 